\title{
S Bosons and Dark Particles of Space Field
}

\author{
Yougang Feng \\ College of Physics, Guizhou University, Guiyang, China \\ Email:ygfeng45@aliyun.com
}

Received 19 July 2016; accepted 21 August 2016; published 24 August 2016

Copyright (C) 2016 by author and Scientific Research Publishing Inc.

This work is licensed under the Creative Commons Attribution International License (CC BY).

http://creativecommons.org/licenses/by/4.0/

(c) (i) Open Access

\section{Abstract}

The spacetime lattice model involves time lattice (static lattice) model and space lattice (dynamic lattice) model, both of which have the same lattices' domains and the same fractal structures. The behaviors of the space field obey the uncertainty relations, which gauge invariance shows the space field is a gauge field, making the electromagnetic field, gravitowagnetic field and the fermion field be gauged, and the Lorentz condition and Lorentz gauge are the intrinsic attributes of the spacetime. The quantization of the classical space field produces $S$ bosons of spin-1, which stimulated states by charges and masses are respectively photons and gravitons. The $S$ bosons in thermal excitation are immeasurable and their energies may be dark. The principle of partition of independent freedom degrees regularizes the degrees for all particles including neutrino, which must have mass. By the $S$ bosons, we interpret newly the virtual photons. Using the spacetime lattice model, we investigate the breaking of the symmetry of the gradient fields and the symmetry of the curl fields for the potential functions of the space field, and the creations and the annihilations of the dark photons and the dark gravitons. The complexity requires us to rename the electroweak phase transition as electro-gravito-weak phase transition. Finally, antiparticles are discussed. Our approach for the lattice models is a kind of renormalization group theory, signifying the breaking of symmetries can be renormalized.

\section{Keywords}

Space, $\mathbf{S}$ Boson, Dark, Lorentz, Freedom Degree, Higgs

\section{Introduction}

In February 2016 scientists announced that they detected gravitational waves by observation of a binary black hole merger [1]. Einstein general relativistic theory and the quantization of the waves become once more hot topic. How should we think about the geometry of spacetime? In our opinion, the framework of Newton's mechanics refers two basic concepts: 1) Absolute space and absolute time; 2) For an object, we can know it's accurate mechanical parameters such as position, momentum, and moment by solving motion equation, i.e., any 
one metric space can provide us infinitely accurate observed information about these parameters. Einstein's special relativistic theory denied Newton's first concept: there is no such thing as either absolute space or absolute time, and there exists only the mixture of the space and the time. However, his general relativistic theory coincides exactly with Newton's second concept. He imagined that if two persons fell downward freely, they wouldn't feel their own weights, and couldn't discern whether they were falling together toward, as if they were in a stationary state, and their freely falling reference frame was in a universe without gravity. Such equivalence description neglects the interaction of the individualities, thus all quantum effects of particles have been abandoned as the mass of a person is far greater than a particle's. There is now a logical paradox: on one hand, the interaction between particles arising from their masses is absolutely eliminated by theorists; on the other hand the gravitons as a type of particle relating to the masses and coming from the quantization of the general relativistic theory are invariably examined by experiments. Yet, on contrary to Einstein' theory, Maxwell theory can represent the electromagnetic field produced by charged particles, in favor of its quantization.

Weak interaction doesn't mean no interaction. To omit weak gravity will thwart our investigation of the interacting forms originated from masses. Einstein argued that spacetime should be warped out of gravity before the establishment of Maxwell laws of electromagnetism, and he spent most of his last 25 years to unify vainly his theory with Maxwell's. It can be understood that the evolution of universe was not well enough known in the 1900 s and early 1905s. According to the standard model of today [2]-[5], mass has no priority to determine the property of spacetime, which is also proved in this paper. We may treat the spacetime as flat like what Maxwell did. To accept this point of view doesn't say we must turn back to the framework of Newton's mechanics. We notice that there exists unique space field together with unique spacetime. No matter whether for electromagnetic force or for gravity, their corresponding ground states are commonly the ground states of the space field. In this sense, the electromagnetic field and the gravitational field all are the stimulated states of the space field. Furthermore, mass not only evokes gravitational field, but also generates wagnetic field. A moving object with mass will be exerted by Lorentz-force-like in the wagnetic field [6].

The discovery of $3 \mathrm{~K}$ microwave background radiation makes us realize there was an early universe as explained by the standard model [7] [8]. The model indicates that the universe has undergone a thermal equilibrium state, which allows us to determine a list of elements. In fact, no one was able by himself to watch such natural scene of the early universe. It is impossible that there is an ideal model to illustrate completely universe, and applicable simplification should be done. For this reason, the standard model has been believed to be an excellent approach to nature so far.

Based on the model, we set up time lattice model and studied on time phase transition, the quanta of the time field are just Higgs with masses, they and the net charges occur simultaneously after the time phase transition [9]. There are two kinds of spacetime, one is mathematical, including nothing. Another is of physics, having its dual space: momentum-energy. We cannot mention the time phase transition for the mathematical one, where time direction is globally and locally allowed to be symmetry. Born out of the universe expansion, a physical space itself, even more than the mathematical one, have to be full of energies, heat diffusion, and temperature distribution. We proved the uncertainty relations are the intrinsic attributes of the spacetime [10]. The relations arise from the duality of the natural bases of the relevant spaces. In terms of mathematics, the inner product of the dual bases is always constant, no matter for which coordinate system.

A stable physical space should have a constant density of energies. This is in fact a dynamic stability as the energies fluctuations, which were proved by the detection of microwave radiation background [11]-[13]. Considering this situation, we construct a physical space lattice model making up of infinite minimal spaces with nonzero volumes due to the uncertainty principle. We put a lattice in each minimal space to represent it. The alternation of the energies can be thought of as the change in the space volume keeping constant density. Such change is equivalent to that the fluctuation force drives the lattices to wiggle around their equilibrium positions, while the space is rigid. Namely, all of the minimal spaces have the identical volumes, and the lattices are successively oscillating. Their equilibrium sites can serve as the coordinate lattices, which determine the fractal structure of the spacetime [14]. When time is in global ordered state, the lattices states cooperate to form harmonic waves, called space fields, and its quantization gives massless bosons, called $\mathrm{S}$ bosons. There are four types of S bosons, corresponding respectively to the four types of Higgs particles [9]. The formula

$c^{2} \mathrm{~d} t^{2}-\mathrm{d} x^{2}-\mathrm{d} y^{2}-\mathrm{d} z^{2}=0$ itself shows the spacetime gauge property, and the light speed $c$ should be essential attributes for the physical spacetime, which can be understood from two hands: On one hand, the motion trail of a stationary spacetime point in the general relativistic theory is the world line with $c t$. On the other hand, the re- 
levant space point is just the equilibrium position of the lattices of the space lattice model. The two descriptions should be equivalent. This implies that if there is no photon, the real moving objector is the waves of the space field, producing the $\mathrm{S}$ bosons that propagate at light speed. In other word, the velocity of the $\mathrm{S}$ bosons is the light speed. Furthermore, the wave rate does be the rate with respect to the lattice equilibrium sites, which are also the coordinate positions for all inertial reference frames, such that the space field waves velocity, i.e. the light speed, is constant for all inertial system.

In Section 2, we prove at first that the Lorentz transformation can't change the uncertainty relations, then introduce classical space field, which quantization leads to $\mathrm{S}$ bosons. By the $\mathrm{S}$ bosons, we explain newly the virtual photons of the quantum electromagnetics. In Section 3, we propose the principle of partition of independent freedom degrees, it regularizes the degrees for all particles. We reveal the space field makes the conventional electromagnetic field and the fermion field be gauged, and the Lorentz condition and the Lorentz gauge are the intrinsic attributes of the spcetime. Combining the time field, we discuss the creations and the annihilations of some quantum fields, there exists dark photons and dark gravitons. Finally, antiparticles are discussed. Section 4 is conclusion remark.

The parameters applied in this paper have the same meanings and definitions as that of the conventional quantum field theory, if there is no particular expatiation.

\section{Theory}

\subsection{The Gauge Invariance of Uncertainty Relations}

Suppose: 1. The Cartesian coordinate systems used by the observes K and K' are such that the axes of one are to parallel to the relevant axes of the others, and 2. Their relative motion is confined in the direction of one of the axes, notate the $\mathrm{x}$-(or $\mathrm{x}^{\prime}$-)axis, the velocity of $\mathrm{K}^{\prime}$ with respect to $\mathrm{K}$ being equal $u$. By Lorentz transformation $x=\left(x^{\prime}+u t^{\prime}\right) / \sqrt{1-u^{2} / c^{2}}, t=\left[t^{\prime}+\left(u / c^{2}\right) x^{\prime}\right] / \sqrt{1-u^{2} / c^{2}}$, and

operators' transformation $\partial / \partial x=\left(\partial / \partial x^{\prime}\right) \mathrm{d} x^{\prime} / \mathrm{d} x+\left(\partial / \partial t^{\prime}\right) \mathrm{d} t^{\prime} / \mathrm{d} x$, we get differential expressions

$$
\mathrm{d} x=\frac{1}{\sqrt{1-u^{2} / c^{2}}}\left(\mathrm{~d} x^{\prime}+u \mathrm{~d} t^{\prime}\right), \frac{\partial}{\partial x}=\frac{1}{\sqrt{1-u^{2} / c^{2}}}\left(\frac{\partial}{\partial x^{\prime}}-\frac{u}{c^{2}} \frac{\partial}{\partial t^{\prime}}\right)
$$

Similarly,

$$
\mathrm{d} t=\frac{1}{\sqrt{1-u^{2} / c^{2}}}\left(\mathrm{~d} t^{\prime}+\frac{u}{c^{2}} \mathrm{~d} x^{\prime}\right), \frac{\partial}{\partial t}=\frac{1}{\sqrt{1-u^{2} / c^{2}}}\left(\frac{\partial}{\partial t^{\prime}}-u \frac{\partial}{\partial x^{\prime}}\right)
$$

From (1) and (2), we have

$$
\frac{\partial}{\partial x} \mathrm{~d} x=\frac{1}{1-u^{2} / c^{2}}\left(\frac{\partial}{\partial x^{\prime}} \mathrm{d} x^{\prime}-\frac{u}{c^{2}} \frac{\partial}{\partial t^{\prime}} \mathrm{d} x^{\prime}+u \frac{\partial}{\partial x^{\prime}} \mathrm{d} t^{\prime}-\frac{u^{2}}{c^{2}} \frac{\partial}{\partial t^{\prime}} \mathrm{d} t^{\prime}\right)
$$

In terms of mathematics, the duality and the orthogonality of the natural bases are

$$
\frac{\partial}{\partial x^{\prime}} \mathrm{d} x^{\prime}=\frac{\partial}{\partial t^{\prime}} \mathrm{d} t^{\prime}=1, \frac{\partial}{\partial x^{\prime}} \mathrm{d} t^{\prime}=\frac{\partial}{\partial t^{\prime}} \mathrm{d} x^{\prime}=0
$$

By Equation (4), Equation (3) turns into

$$
\frac{\partial}{\partial x} \mathrm{~d} x=\frac{1}{1-u^{2} / c^{2}}\left(1-u^{2} / c^{2}\right)=1
$$

Namely,

$$
\frac{\partial}{\partial x} \mathrm{~d} x=\frac{\partial}{\partial x^{\prime}} \mathrm{d} x^{\prime}=1
$$

With the same reason, we obtain

$$
\frac{\partial}{\partial t} \mathrm{~d} t=\frac{\partial}{\partial t^{\prime}} \mathrm{d} t^{\prime}=1
$$


We can also get the same proof, exchanging $u, x^{\prime}$, and $t^{\prime}$ for $-u, x$, and $t$, respectively in the above equations due to the relativity. According to reference [10], the uncertainty relation depends on the duality of the natural bases, Equations (6) and (7) show that Lorentz transformation can't change the uncertainty relations, i.e., the behavior of the space field obeys the same law in different inertial reference frames. In a word, Lorentz gauge is the intrinsic attribute of the spacetime.

\subsection{Classical Space Field}

The gradient fields of the potential functions of the space field are symmetric, $\pm \varphi \pm \partial A / \partial t$; the curl fields are also symmetric, $\pm \nabla \times \boldsymbol{A}$; where $\varphi$ is the scalar potential, and $\boldsymbol{A}$ the vector potential. It seems as if there were symmetric potential functions $\pm \varphi$ and $\pm \boldsymbol{A}$ [6], that is not true at all! The potential functions are unique, $\varphi$ and $\boldsymbol{A}$. The total intensity of each kind of the fields is zero because of the symmetry. As the following expressed, the potential functions satisfy wave equations, which solutions are traveling waves at light speed. Let $\boldsymbol{\Omega}=\boldsymbol{\Omega}_{1}+\boldsymbol{\Omega}_{2}$ denote the gradient field, $\boldsymbol{\Theta}=\boldsymbol{\Theta}_{1}+\boldsymbol{\Theta}_{2}$ the curl field. The symmetric fields are successively

$$
\begin{gathered}
\boldsymbol{\Omega}_{1}=-\nabla \varphi-\frac{1}{c} \frac{\partial \boldsymbol{A}}{\partial t}, \quad \boldsymbol{\Omega}_{2}=\nabla \varphi+\frac{1}{c} \frac{\partial \boldsymbol{A}}{\partial t} \\
\boldsymbol{\Theta}_{1}=\nabla \times \boldsymbol{A}, \quad \nabla \times \boldsymbol{\Theta}_{1}=\frac{1}{c} \frac{\partial \boldsymbol{\Omega}_{1}}{\partial t}+\frac{4 \pi}{c} \boldsymbol{j}_{1} \\
\boldsymbol{\Theta}_{2}=-\nabla \times \boldsymbol{A}, \quad \nabla \times \boldsymbol{\Theta}_{2}=\frac{1}{c} \frac{\partial \boldsymbol{\Omega}_{2}}{\partial t}+\frac{4 \pi}{c} \boldsymbol{j}_{2} \\
\nabla \times \boldsymbol{\Omega}_{1}=-\frac{1}{c} \frac{\partial \boldsymbol{\Theta}_{1}}{\partial t}, \quad \nabla \times \boldsymbol{\Omega}_{2}=-\frac{1}{c} \frac{\partial \boldsymbol{\Theta}_{2}}{\partial t} \\
\nabla \cdot \boldsymbol{\Omega}_{1}=\nabla \cdot \boldsymbol{\Omega}_{2}=0 \quad \text { (passive fields) }
\end{gathered}
$$

Since there is neither charge nor mass, so $\boldsymbol{j}_{1}=\boldsymbol{j}_{2}=0$. By (8) and (12), we get

$$
\nabla^{2} \varphi-\frac{1}{c^{2}} \frac{\partial^{2} \varphi}{\partial t^{2}}+\frac{1}{c} \frac{\partial}{\partial t}\left(\nabla \cdot A+\frac{1}{c} \frac{\partial \varphi}{\partial t}\right)=0
$$

For the space field

$$
\nabla^{2} \varphi-\frac{1}{c^{2}} \frac{\partial^{2} \varphi}{\partial t^{2}}=0
$$

Equation (13.2) is a hyperbolic differential equation, describing the wave of the space field. Equations (13.1) and (13.2) result in Lorentz condition

$$
\nabla \cdot A+\frac{1}{c} \frac{\partial \varphi}{\partial t}=0
$$

Equation (14) manifests the Lorentz condition is the intrinsic attribute of the spacetime. From (9)

$$
\nabla \times(\nabla \times \boldsymbol{A})=\frac{1}{c} \frac{\partial \mathbf{\Omega}_{1}}{\partial t}
$$

Using the algebraic transformation, the left hand of (15) becomes $-\nabla^{2} \boldsymbol{A}+\nabla(\nabla \cdot \boldsymbol{A})$, combining (10), we change (8) into

$$
-\nabla^{2} \boldsymbol{A}+\frac{1}{c^{2}} \frac{\partial^{2} \boldsymbol{A}}{\partial t^{2}}+\nabla\left(\nabla \cdot \boldsymbol{A}+\frac{1}{c} \frac{\partial \varphi}{\partial t}\right)=0
$$

Using (14) and (16.1), we obtain

$$
\nabla^{2} \boldsymbol{A}-\frac{1}{c^{2}} \frac{\partial^{2} \boldsymbol{A}}{\partial t^{2}}=0
$$

The meaning of (16.2) is analogue to that of (13.2). For $\boldsymbol{\Omega}_{2}$ and $\boldsymbol{\Theta}_{2}$, we can derive the same consequences 
as (13.2) and (16.2).

\subsection{The Quantization of the Space Field}

There is no current since there is neither electricity charge nor mass such that the equation of the space field behaves as

$$
\partial_{\mu} \partial^{\mu} A_{v}=0
$$

where the derivative operator $\partial_{\mu}=\partial / \partial x^{\mu}=\left(\partial / \partial x^{0}, \nabla\right), \hbar=c=1, \quad E=P^{0}=P_{0}, P_{\mu}=i \partial / \partial x^{\mu}, A^{\mu}=(\varphi, \boldsymbol{A})$, $\mu=0,1,2,3$, corresponding to $t, x, y, z$, respectively. Since the wave propagates at light speed there is no inertial mass, the equation is like in form Klein-Gorden (K-G) field equation with mass $m=0$. Applying mechanically the solution of the K-G equation, we express $A_{\mu}$ as

$$
A_{\mu}=\int \mathrm{d}^{3} k \frac{1}{\sqrt{(2 \pi)^{3} 2 k}}\left[a_{\mu}(\boldsymbol{k}) \mathrm{e}^{-i k x}+a_{\mu}^{+}(\boldsymbol{k}) \mathrm{e}^{i k x}\right]
$$

where the wave vector is $k_{0}=\omega_{k}=k$. The commutation relations for the creators and the annihilators are

$$
\begin{aligned}
& {\left[a_{j}(\boldsymbol{k}), a_{j^{\prime}}^{+}\left(\boldsymbol{k}^{\prime}\right)\right]=\delta_{j j^{\prime}} \delta^{3}\left(\boldsymbol{k}-\boldsymbol{k}^{\prime}\right),} \\
& {\left[a_{0}(\boldsymbol{k}), a_{0}^{+}\left(\boldsymbol{k}^{\prime}\right)\right]=-\delta^{3}\left(\boldsymbol{k}-\boldsymbol{k}^{\prime}\right),} \\
& {\left[a_{j}(\boldsymbol{k}), a_{0}^{+}\left(\boldsymbol{k}^{\prime}\right)\right]=[a, a]=\left[a^{+}, a^{+}\right]=0}
\end{aligned}
$$

Adjusting the coordinates, we introduce new operators: $a^{\mu}(\boldsymbol{k})=\varepsilon^{\mu}(\boldsymbol{k}, \lambda) a(\boldsymbol{k}, \lambda),(\lambda=0,1,2,3)$, where $\varepsilon^{\mu}(\boldsymbol{k}, \lambda)$ is unit vector, $\boldsymbol{k}$ is parallel to the $z$-axis and perpendicular to the $x$-, $y$-axes. Since the Lorentz condition and the field action are invariant for the special relativistic theory, we have

$$
\begin{aligned}
& {\left[a(\boldsymbol{k}, j), a^{+}\left(\boldsymbol{k}^{\prime}, j\right)\right]=\delta^{3}\left(\boldsymbol{k}-\boldsymbol{k}^{\prime}\right), j=1,2,3} \\
& {\left[a(\boldsymbol{k}, 0), a^{+}\left(\boldsymbol{k}^{\prime}, 0\right)\right]=-\delta^{3}\left(\boldsymbol{k}-\boldsymbol{k}^{\prime}\right)}
\end{aligned}
$$

Other commuting operators go away. Equation (18) then translates into

$$
A_{\mu}(x)=\sum_{\lambda} A_{\mu}^{\lambda}(x)=\sum_{\lambda} \int \mathrm{d}^{3} k \frac{1}{\sqrt{(2 \pi)^{3} 2 k}} \varepsilon_{\mu}(\boldsymbol{k}, \lambda)\left[a(\boldsymbol{k}, \lambda) \mathrm{e}^{-i k x}+a^{+}(\boldsymbol{k}, \lambda) \mathrm{e}^{i k x}\right]
$$

If $\lambda=1$ or $2, A_{\mu}^{\lambda}(x)$ obeys Lorentz condition, $\partial^{\mu} A_{\mu}^{\lambda}=0$, and $k^{\mu} \varepsilon_{\mu}(\boldsymbol{k}, \lambda)=0$. If $\lambda=0$ or $3, A_{\mu}^{\lambda}(x)$ violates the condition, so the $\mathrm{S}$ bosons are transversal particles of spin-1.

\subsection{Stimulated States and Virtual Photons}

The properties of the stimulated fields are described by reference [6]: Electricity charges stimulate the space field to produce electromagnetic field, which quanta are photons carrying electromagnetic forces. Masses evoke gravitowagnetic field, which quanta are gravitons carrying the gravitowagnetic forces. Besides the charges and masses, heat also can make $\mathrm{S}$ bosons be in the states of higher energies than the ground states'. The intensities of the total gradient field and the total curl field of the potential functions vanish still, if there is only the thermal excitation. The $\mathrm{S}$ bosons, except photons and gravitons, therefore, even though being the thermal stimulated states, don't observably carry force such as the electromagnetic force or the gravitowagnetic force. In this sense, the $\mathrm{S}$ bosons can't be directly measured, and their energies maybe dark. The total energy $E$ of the field is

$$
E=\sum_{\omega}\left(n_{\omega}+1 / 2\right) \hbar \omega
$$

where $\hbar$ is Plank constant, $\omega$ angle frequency, $n_{\omega}$ the number of S bosons with frequency $\omega,(1 / 2) \hbar \omega$ the ground state energy. The average number of the $n_{\omega},\left\langle n_{\omega}\right\rangle$, is determined by Plank distribution function

$$
\left\langle n_{\omega}\right\rangle=1 /\left[\exp \left(\hbar \omega / k_{B} T\right)-1\right]
$$

Introducing the concept of S bosons, the interaction between electrons can be newly interpreted as the fol- 
lowing: The first electron $e_{1}$ stimulates a $\mathrm{S}$ boson making it become the first photon $q_{1}$, the $e_{1}$ then loses its partial energy, denoted by $e_{1}(-)$

$$
e_{1}+S \rightarrow q_{1}+e_{1}(-)
$$

Similarly, for the second electron $e_{2}$ and the second photon $q_{2}$,

$$
e_{2}+S \rightarrow q_{2}+e_{2}(-)
$$

The $e_{1}(-)$ and the $q_{2}$ exchange energy, the $q_{2}$ loses energy and turns back to be $\mathrm{S}$ boson again. The $e_{1}(-)$ obtains energy to become $e_{1}^{\prime}$,

$$
e_{1}(-)+q_{2} \rightarrow S+e_{1}^{\prime}
$$

For the $e_{2}(-)$, the same process is

$$
e_{2}(-)+q_{1} \rightarrow S+e_{2}^{\prime}
$$

where the $q_{1}$ and $q_{2}$ are just the virtual photons.

\section{Discussion}

\subsection{The Principle of Partition of Independent Freedom Degrees}

The world we are living in is a global ordered-time spacetime after the electroweak phase transition, while the fractal structures disappear. Different from those spaces of extra dimensions [15] [16], the fractal structures of higher dimensions can return to the 4-dimensional spacetime by the scaling law [14]. The fractal dimensions used in the lattice models are called box-counting dimensions, generally defined as [17]

$$
D=\lim _{\delta \rightarrow 0} \frac{\log N_{\delta}(F)}{-\log \delta}
$$

where $F$ is any non-empty bounded subset of $R^{n}$ and $N_{\delta}(F)$ is the smallest number of sets of diameter at most $\delta$, which can cover $F$. For a lattice model the $N_{\delta}(F)$ is the total number of the lattices' domains in a sub-block [14]. For the self-similar transformations the $D$ is just the similarity dimension. The scaling law regularizes a lattice domain to be an open set of one unit length diameter. These regularities are still effective after the electroweak phase transition. There are two types of lattices in each domain: dynamic lattice and static lattice, the latter positions at the former equilibrium site. The static lattice model is insisted of the static lattices, the dynamic lattice model is made up of the dynamic lattices. The dynamic lattice model can draw the space field, which acts as a background field for the other fields being applicable to the static lattice model. For this reason, we also call the dynamic lattice model as the space lattice model. The two kinds of models correspond to the same spacetime because of the dimension definition. Let coordinates $x^{\prime}$ denote the dynamic lattices, $x$ do the static lattices. When there is not any other field, the potential functions $A\left(x^{\prime}\right)$ and $A(x)$ are identical for the space field (see subsection 2.2).

The principle of partition of independent freedom degrees is that there are $(D+2)$ freedom degrees to be partitioned in the $(D+1)$-dimensional spacetime: $D$ 's space's, one time's, one spin's, $(D+1)$ of which each particle must own. The Higgs particle always occupies independently the time's degree. After the electroweak phase transition the spacetime becomes 4-dimensions $(D=3)$, the Higgs has three spatial degrees for the propagation of its waves. Hence, the Higgs is a scalar boson. The space field lies in the dynamical lattice model different from the static lattice model, in which the time field is set. Because of this, a S boson is independent of the time's degree and has one spin's degree, besides three spatial degrees. Other particles are accommodated to the static lattice model, and each of them has three spatial degrees and one spin degree. Each of them is forced to couple with the Higss particles to share (not independently) the time's degree, which causes masses. According to this principle a neutrino or an antineutrino must have mass, no matter how weak their coupling with the Higgs may be. Photons and gravitons as the stimulated states of the S bosons have the same degrees as the $\mathrm{S}$ bosons'.

\subsection{The Nature of the Lorentz Gauge}

The so-called conventional electromagnetic field is the field stimulated by charges, which can be describe by 
Maxwell theory. When the space field isn't omitted the potential function of the electromagnetic field is assigned as $A_{\mu}\left(x^{\prime}\right)$, and the potential function of the conventional electromagnetic field as $A_{\mu}(x)$, while the thermal stimulated state of space field is neglected. It should be emphasized that only the $A_{\mu}(x)$ relates to the electricity current. As the field stimulated by charge is far stronger than the thermal stimulated space field, we expand the $A_{\mu}\left(x^{\prime}\right)$ in a series around the $x$, taking its linear term,

$$
A_{\mu}\left(x^{\prime}\right)=A_{\mu}(x)+\left[\partial^{\mu} A_{\mu}\left(x^{\prime}\right)\right]_{x^{\prime}=x} \cdot\left(x^{\prime}-x\right)
$$

Only the second expansion term relates to the thermal stimulated space field independent of the current. Recall the definition of the derivative of function $f(x)$ at the $x$

$$
\frac{\mathrm{d} f(x)}{\mathrm{d} x}=\lim _{\Delta x \rightarrow 0} \frac{f(x+\Delta x)-f(x)}{\Delta x}
$$

Clearly, the points $x+\Delta x$ and $x$ are in the same domain if $\Delta x \rightarrow 0$ but zero. This admits $\left[\partial^{\mu} A_{\mu}\left(x^{\prime}\right)\right]_{x^{\prime}=x}=\partial^{\mu} A_{\mu}(x)$. Since the scaling law defines the distance of nearest neighbors as one unit length [14], thus $0<\left|x^{\prime}-x\right|<1 / 2$. We make the domain be so small that the following approximate expression holds

$$
A_{\mu}\left(x^{\prime}\right) \cong A_{\mu}(x)+\partial^{\mu} A_{\mu}(x) \cdot \alpha=A_{\mu}(x)+\partial^{\mu}\left[\alpha \cdot A_{\mu}(x)\right]
$$

where $\alpha$ is constant and $0<|\alpha|<1 / 2$. For the conventional electromagnetic field, the Lorentz gauge transformation is

$$
A_{\mu}^{\prime}(x)=A_{\mu}(x)+\partial^{\mu} \theta(x)
$$

Equations (31) and (32) say the same field, hence, we have

$$
A_{\mu}^{\prime}(x)=A_{\mu}\left(x^{\prime}\right)
$$

Here $A_{\mu}^{\prime}(x)$ is the potential function including the conventional electromagnetic field and the thermal stimulated space field. Moreover, from (31) and (32):

$$
\partial^{\mu} \theta(x)=\partial^{\mu}\left[\alpha \cdot A_{\mu}(x)\right]=\alpha \cdot \partial^{\mu} A_{\mu}(x)
$$

where $\partial^{\mu} A_{\mu}(x)$ is the derivative of the $A_{\mu}\left(x^{\prime}\right)$ with respective to the $x^{\prime}$ at the point $x^{\prime}=x$. The second derivative of the $A_{\mu}\left(x^{\prime}\right)$ at the $x$ is

$$
\left[\partial_{\mu} \partial^{\mu} A_{\mu}\left(x^{\prime}\right)\right]_{x^{\prime}=x}=\partial_{\mu} \partial^{\mu} A_{\mu}(x)
$$

Since only the $A_{\mu}(x)$ of the first term of Equation (32), which is the potential function of the conventional electromagnetic field, refers to the current, the $A_{\mu}(x)$ in this derivative term should be regarded as the potential function of the thermal stimulated space field (the $x$ is the equilibrium position of the $x^{\prime}$, so the $x$ can be viewed as a special point of the $x^{\prime}$ system; inversely, the $x^{\prime}$ can't belong to the $x$ system relating only to the equilibrium sites), so Equation (35) is the wave equation of the space field similar to Equations (13.2) and (16.2). From Equations (34) and (35), we get

$$
\partial_{\mu} \partial^{\mu} \theta(x)=0
$$

This is just the Lorentz gauge transformation for the conventional electromagnetic field. Equations (32) and (36) result in the Lorentz condition

$$
\partial^{\mu} A_{\mu}^{\prime}(x)=\partial^{\mu} A_{\mu}(x)=0
$$

We understand now that the Lorentz gauge is just the intrinsic attribute of the spacetime rather than an artificial additional condition. In addition, the $\theta(x)$ can also describe the space field because of its linear relation with the $A_{\mu}(x)$.

The space field behaves in the way of the symmetry $\mathrm{U}(1)$, and its action on a fermion field $\Psi$ is represented as

$$
\Psi^{\prime}=\mathrm{e}^{-i Q e \theta(x)} \Psi
$$


where $Q e$ is the electricity charge of the fermion, Equation (38) is called the gauge transformation of the fermion field. The space field guarantees that the interaction form remains the same when the fermion field takes different phase factors at different spacetime positions. Hence, we should consider the space field as Lorentz gauge field. It is the space field that makes the conventional electromagnetic field and the fermion field become gauge fields. With the same reason, the gravitowagnetic field is also a gauge field.

\subsection{Dark Photons and Dark Gravitons}

The critical temperature $T_{c}$ of the time phase transition is given by reference [9], which shows if the temperature $T$ is higher than $T_{c}$, the particles number equals the antiparticles number, the total charge is neutral, so there is no stimulated field by the charges. When time becomes global ordered, in the temperature region $T_{e w}<T \leq T_{c}, T_{e w}$ is the turning point of the electroweak phase transition, the particles number attains ascendant and four types of time fields (Higgs particles) emerge, accompanying four types of space fields with the same fractal structures as the time fields'. Thus, the symmetry of the gradient fields and the symmetry of the curl fields are together broken, the net charges and the masses stimulate simultaneously and separately four types electromagnetic fields and four types of gravitowagnetic fields [6]. The photons born in the inner space of a sub-block or a block, concern only short-range interactions, they may be the dark photons [18] [19]. The gravitons produced in the same inner spaces have only limited interacting range, and may be called dark gravitons. The $D$-dimensional $(2<D<3)$ inner space of a sub-block and the $D^{4}$-dimensional $\left(16<D^{4}<81\right)$ inner space of a block defer from the 4-dimensional spacetime, corresponding separately to the spacetime of $(D+1)$ dimensions and the spacetime of $\left(D^{4}+1\right)$ dimensions, while the Lorentz gauge aren't available. The subsequent symmetries may acquire new gauge fields bring about new particles. At the same time, the symmetry $\mathrm{SU}(2)$ in the fractal structures generates particles $W^{+}, W^{-}$, and $Z$. All fields are of localities due to the sizes of the sub-blocks and the blocks are confined. We don't exclude the dark photons and the dark gravitons participate in the interactions among the particles such as $W^{+}, W^{-}, Z, e^{+}$, and $e^{-}$or other unknown particles. When $T \leq T_{e w}$ there are no fractal structures with the disappearing of the sub-blocks and the blocks, the correlation lengths of the lattices states become infinity. Three types of Hggis particles, three types of photons (dark photons), and three types of gravitons (dark gravitons) are annihilated, the rest, the fourth types of them become new types: the Higgs particles lead to masses [9], the gravitowagnetic field together with the electromagnetic field form in the 4-dimensional spacetime. Their quanta, the gravitons and the photons, possess infinite forces ranges, the dark photons and the dark gravitons turn possibly into electrons and positrons in their annihilation process. In this sense, we may rename the transition as electro-gravito-weak phase transition.

\subsection{Antiparticles}

For the time field, the p-time state relates to particles, the n-time state to antiparticles [9]. Since the space field is independent of the time field, a S boson is just its antiparticle, so do the photon and the graviton. Conversely, an antineutrino and a neutrino can be distinguishable from one another as they rely on the time field.

\section{Conclusion}

We persist that the solutions for all of phenomena can be found in the spacetime. The quantization of the spacetime gives rise to the Higgs particles and the $\mathrm{S}$ bosons, which excitation states relate to dark photons, photons, dark gravitons, and gravitons. The symmetric characteristics of the spacetime lead to a series of gauge fields, illustrating the intrinsic attributes of the spacetime. The spacetime lattice model contains the time lattice model and the space lattice model, and its fractal structures provide us a chance to discover new symmetries, introducing new gauge fields and chasing new particles.

\section{References}

[1] Abbott, B.P., et al. (2016) Physical Review Letters, 116, Article ID: 061102. http://dx.doi.org/10.1103/PhysRevLett.116.061102

[2] Glashow, S.L. (1961) Nuclear Physics, 22, 579-588. http://dx.doi.org/10.1016/0029-5582(61)90469-2

[3] Weinberg, S. (1967) Physical Review Letters, 19, 1264-1266. http://dx.doi.org/10.1103/PhysRevLett.19.1264

[4] Salam, A. (1979) Nobel Lecture Physics, 513-538. 
http://www.nobelprize.org/nobel prizes/physics/laureates/1979/salam-lecture.pdf

[5] Higgs, P.W. (1964) Physical Review Letters, 13, 508-509. http://dx.doi.org/10.1103/PhysRevLett.13.508

[6] Feng, Y.G. (2015) Journal of ModernPhysics, 6, 573-577. http://dx.doi.org/10.4236/jmp.2015.65062

[7] Penzias, A.A. (1978) Nobel Lecture, 444-457. http://www.nobelprize.org/nobel_prizes/physics/laureates/1978/penzias-lecture.pdf

[8] Wilson, R.W. (1978) Nobel Lecture, 463-483. http://www.nobelprize.org/nobel_prizes/physics/laureates/1978/wilson-lecture.pdf

[9] Feng, Y.G. (2016) Journal of Modern Physics, 7, 536-542. http://dx.doi.org/10.4236/jmp.2016.76056

[10] Feng, Y.G. (2014) Natural Science, 6, 1149-1158. http://dx.doi.org/10.4236/ns.2014.614103

[11] Subrahmanyan, R. (1997) Journal of Astrophysics \& Astronomy, 18, 251-255. http://dx.doi.org/10.1007/BF02709314

[12] Mather, J.C. (2006) Nobel Lecture, 64-96. www.nobelprize.org/nobel prizes/physics/laureates/2006/mather lecture.pdf

[13] Smoot, G.F. (2006) Nobel Lecture, 113-166. http://www.nobelprize.org/nobel_prizes/physics/laureates/2006/smoot_lecture.pdf

[14] Feng, Y.-G. (2014) American Journal of Modern Physics, 3, 184-194. http://dx.doi.org/10.11648/j.ajmp.20140304.16

[15] Hewett, J. and Spiropulu, M. (2002) Annual Review of Nuclear and Particles Science, 52, 397-424. http://dx.doi.org/10.1146/annurev.nucl.52.050102.090706

[16] Holman, M. (2012) Physical Degrees of Freedom in Higgs Models. http://arxiv.org/abs/0910.4054

[17] Falconer, K. (2003) Fractal Geometry. 2nd Edition, John Wiley \&Sons, London.

[18] Krasznahorkay, A.J., et al. (2016) Physical Review Letters, 116, Article ID: 042501, http://dx.doi.org/10.1103/PhysRevLett.116.042501

[19] Feng, J.L., et al. (2016) Evidence for a Protophobic Fifth Force from ${ }^{8}$ Be Nuclear Transitions. http://arxiv.org/abs/1604.07411

\section{Submit or recommend next manuscript to SCIRP and we will provide best service for you:}

Accepting pre-submission inquiries through Email, Facebook, LinkedIn, Twitter, etc.

A wide selection of journals (inclusive of 9 subjects, more than 200 journals)

Providing 24-hour high-quality service

User-friendly online submission system

Fair and swift peer-review system

Efficient typesetting and proofreading procedure

Display of the result of downloads and visits, as well as the number of cited articles

Maximum dissemination of your research work

Submit your manuscript at: http://papersubmission.scirp.org/ 